\title{
$\mathrm{M}|\mathrm{R}| \mathrm{S}$

A Publication of the Materials Research Society

Volume 23, Number 3 ISSN: 0883-7694 CODEN: MRSBEA

MATERIALS

FOR SPORTS

32 Materials for Sports

F.H. Froes, Guest Editor

(with contributions by S. Haake,

S. Fagg, K. Tabeshfar, and X. Velay)

39 Material Matters:

Performance Standards

Governing New Materials

for Sports

J.N. Gelberg

42 Titanium Golf Clubs

C. Shira and F.H. Froes

47 Education Exchange: Independent-Study Course

Examines Materials in

Tennis Rackets and

Hockey Sticks

E.M. Lenoe

50 The Role of Materials in the Design of an Improved Cricket Bat

C. Grant

54 Historical Note:

Bicycles Roll Along from Wooden Wagon Wheel to Alloy Tubing

C.J. McMahon, Jr.

56 Modeling Bicycle-Rider Vibrations: Implications for Materials Selection

T.T. Gribb, F.J. Worzala, and G.W. Gribb

\section{MRS NEWS}

591997 Fall Meeting Reflects Popularity and Breadth of MRS Meetings

76 Charles B. Duke Receives 1997 Woody Award

76 MRS Invites Nominations for the Von Hippel Award, Turnbull Lectureship, and MRS Medal

78 Congressional Fellow Brian Holloway Illuminates Materials Issues in Washington Policy

79 Anne Mayes Named Outstanding Young Investigator for Work on Macromolecules

80 Plenary Speaker Lockwood to Address Materials Aspect of Volcanoes at MRS Spring Meeting

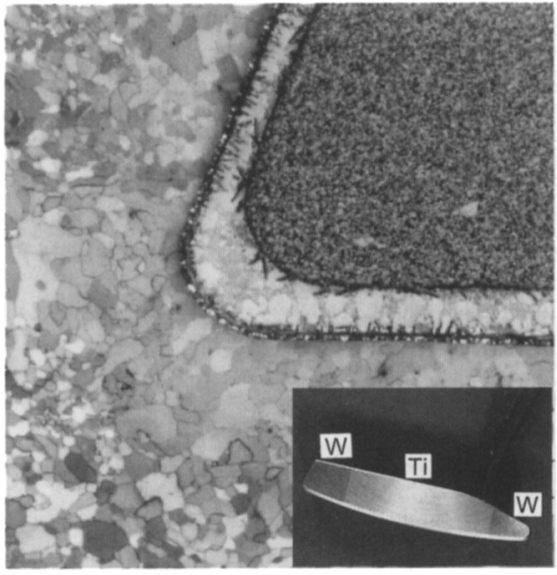

ON THE COVER: Titanium-tungsten braze joint in a putter.

Inset: Putter featuring a titanium center section and tungsten for the heel and toe. The moment of inertia is $4,330 \mathrm{~g} / \mathrm{cm}^{2}$, a susbstantial $65 \%$ increase over earlier putters made of $100 \%$ brass.

\section{ABSTRACTS}

87 Abstracts for May 1998 Journal of Materials Research

\section{DEPARTMENTS}

6 Material Matters

What Are the Options for

Disposition of Excess Weapons

Plutonium?

W. Stoll

18 Letters to the Editor

19 Research/Researchers

27 Washington News

29 Public Affairs Forum

30 Resources

84 Library

Mössbauer Spectroscopy Applied to Magnetism and Materials

Science, Vol. 2, G.J. Long and

F. Grandjean, eds., reviewed by

G. Le Caër; Current Opinion in Solid State and Materials Science,

A.K. Cheetham, M.S. Dresselhaus, and J.M. Thomas, eds., reviewed

by P. Calvert; Forward Recoil

Spectrometry: Applications

to Hydrogen Determination in

Solids, J. Tirira, Y. Serruys, and

P. Trocellier, reviewed by K-P. Lieb

85 Advertisers in This Issue

97 Calendar

101 Classified

104 Posterminaries 


\section{About the Materials Research Society}

The Materials Research Society (MRS), a not-forprofit scientific association founded in 1973, promotes interdisciplinary goal-oriented basic research on materials of technological importance. Membership in the Society includes over 12,000 scientists, engineers, and research managers from industrial, government, and university research laboratories in the United States and nearly 68 countries.

The Society's interdisciplinary approach differs from that of single-discipline professional societies because it promotes information exchange across the many technical fields touching materials development. MRS sponsors two major international anrual meetings encompassing approximately 70 topical symposia, and also sponsors numerous single-topic scientific meetings. The Society recognizes professional and technical excellence and fosters technical interaction in local geographic regions through Sections and University Chapters.

MRS participates in the international arena of materials research through the International Union of Materials Research Societies (IUMRS). MRS is a member of the Federation of Materials Societies and is an affiliate of the American Institute of Physics.

MRS publishes symposium proceedings, MRS Bulletin, Journal of Materials Research, and other publications related to current research activities.

MRS Bulletin (ISSN: 0883-7694) is published 12 times a year by the Materials Research Society, 506 Keystone Drive, Warrendale, PA 15086-7573. Application to mail at periodicals rates has been approved at Warrendale, PA and at additional mailing offices. POSTMASTER: Send address changes to MRS Bulletin in care of the Materials Research Society, at the address listed; phone 724 779-3003; fax 724-779-8313. Printed in the U.S.A.

Additional copies of articles in MRS Bulletin may be made at $\$ 2.50$ per article. This fee can be paid to the Materials Research Society through the Copyright Clearance Center, Inc., 27 Congress Street, Salem, MA 01970.

Membership in MRS is $\$ 75$ annually for regular members, $\$ 25$ for students. Dues include an allocation of $\$ 29$ ( $\$ 17$ for students) to a subscription to MRS Bulletin. Individual member subscriptions are for personal use only. Non-member subscription rates are $\$ 155$ for one calendar year (12 issues) within the U.S.A. and $\$ 215$ elsewhere. Single copies may be purchased for $\$ 16$ each. Send subscription orders to Subscription Department, Materials Research Society, 506 Keystone Drive, Warrendale, PA 15086-7573.

MRS Bulletin is included in Current Contents $/$ Engineering, Computing, and Technology; Current Contents $\% /$ Physical, Chemical, and Earth Sciences, the SciSearch online database, Research Alert ${ }^{*}$, Science Citation Index, and the Materials Science Citation Index ${ }^{\text {Tw. }}$. Back volumes of MRS Bulletin are available in $16 \mathrm{~mm}$ microfilm, $35 \mathrm{~mm}$ microfilm, or $105 \mathrm{~mm}$ microfiche through University Microfilms Inc, 300 North Zeeb Road, Ann Arbor, Michigan 48106.

\section{Materials Research Society 506 Keystone Drive \\ Warrendale, PA 15086-7573 USA \\ Tel. 724-779-3003; Fax 724-779-8313} http://www.mrs.org/

Editorial Office • 506 Keystone Drive • Warrendale, PA 15086-7573 USA

Tel. 724-779-3004 ext. 522; fax 724-779-8313; http://www.mrs.org/

Editor

E.L. Fleischer

Managing Editor

J. Meiksin

Assistant Editor

L.R. Gallagher

Art Director

E. Stiller

Design/Production

T. Aiello and S. Franklin

Editorial Assistants

J. Dininny and

M. Wilmoth

\section{EDITORIAL BOARD}

M.A. Nastasi, Chair

Los Alamos National Laboratory

Los Alamos, NM

W.L. Brown

Bell Laboratories, Lucent Technologies

Murray Hill, NJ

R.W. Cahn (1997 Visiting Scientist)

Cambridge University

Cambridge, UK

D.J. Eaglesham

Bell Laboratories, Lucent Technologies

Murray Hill, NJ

VOLUME ORGANIZERS

1998

0 . Auciello

Argonne National Laboratory

Argonne, IL

\section{9}

M.I. Baraton

University of Limoges

Limoges, France

\section{VISITING SCIENTISTS}

V.S. Arunachalam (1998)

Carnegie Mellon University

Pittsburgh, PA

Advertising

M.E. Kaufold

Circulation

S. Forrest

Guest Editor

F.H. Froes

Special Contributors

S. Beckerman, P. Calvert,

E.N. Kaufmann, G. Le Caër

K-P. Lieb, J.M. Phillips, G. Rao,

L.A. Snyder, and W. Stoll

Special Consultant

M. Goodway
Associate Editor-Europe

I.W. Boyd, University College London Dept. of Electronic and

Electrical Engineering

Torrington Place

London WCI E7 JE, U.K

Tel. 44-171-380-7300 or 7302

Book Review Board

M.A. Nastasi (Chair), J.C. Bravman,

R.W. Cahn, M.L. Green, and

E.J. Kramer

MRS Office of Public Affairs

601 13th Street, NW, Suite 1000 South

Washington, DC 20005-3807

Tel. 202-661-2285, Fax 202-661-2299

R.L. Fleischer (1995)

Union College

Schenectady, NY

E.J. Kramer

Santa Barbara, CA

G.G. Long

National Institute of Standards

and Technology

Gaithersburg, MD

S.C. Moss

Aerospace Corporation

Los Angeles, CA

W.D. Nix

Stanford University

Stanford, CA
University of California-Santa Barbara

S.J. Pearton

University of Florida

Gainesville, FL

S.T. Picraux

Sandia National Laboratories

Albuquerque, NM

$Y$ Shiohara

ISTEC

Tokyo, Japan

C.C. Tsai

Applied Komatsu Technology

Santa Clara, CA

R.J. Composto

University of Pennsylvania

Philadelphia, PA

P.M. Fauchet

University of Rochester

Rochester, NY

R.C. Cammarata

Johns Hopkins University

Baltimore, MD

S.M. Yalisove

University of Michigan

Ann Arbor, MI

R.W. Cahn (1997)

Cambridge University

Cambridge, UK

Los Alamos National Laboratory

Los Alamos, NM

\begin{tabular}{lll}
\hline & 1998 MRS EXECUTWE COMMITEE & \\
\hline Presidént & Treasurer & Councillors \\
R.J. Nemanich & A. Hurd & H.A. Atwater \\
North Carolina State University & Sandia National Laboratories & California Institute of Technology \\
Vice President and President-Elect & Immediate Past President & M.L. Green \\
R. Gibala & R. Hull & Bell Laboratories/Lucent \\
University of Michigan & University of Virginia & Technologies \\
Secretary & & Executive Director \\
C.C. Tsai & & Materials Research Society \\
Applied Komatsu Technology & & John B. Ballance
\end{tabular}

\begin{tabular}{|c|c|c|c|c|c|c|}
\hline \multicolumn{7}{|c|}{ INTERNATIONAL UNION OF MATERIALS RESEARCH SOCIETIES } \\
\hline $\begin{array}{l}\text { President } \\
\text { R.C. Ewing } \\
\text { University of Michigan } \\
\text { Ann Arbor, MI, USA }\end{array}$ & $\begin{array}{l}\text { First Vice President } \\
\text { H-D. Li } \\
\text { Tsinghua University } \\
\text { Beijing, China }\end{array}$ & $\begin{array}{l}\text { Second Vice President } \\
\text { P.A. Glasow } \\
\text { Erlangen, Germany }\end{array}$ & $\begin{array}{l}\text { Secretary } \\
\text { C-G. Li } \\
\text { Beijing, China }\end{array}$ & $\begin{array}{l}\text { Treasurer } \\
\text { R-I. Yamamoto } \\
\text { University of Tokyo } \\
\text { Tokyo, Japan }\end{array}$ & $\begin{array}{l}\text { Immediate Past President } \\
\text { M. Doyama } \\
\text { Nishi-Tokyo University } \\
\text { Tokyo, Japan }\end{array}$ & $\begin{array}{l}\text { General Secretary } \\
\text { R.P.H. Chang } \\
\text { Northwestern University } \\
\text { Evanston, Illinois, USA }\end{array}$ \\
\hline \multicolumn{7}{|c|}{ IUMRS ADHERING BODIES } \\
\hline \multicolumn{2}{|c|}{$\begin{array}{l}\text { Australian Materials Research Society (A-MRS) } \\
\text { J.S. Williams, Australian National University }\end{array}$} & \multicolumn{3}{|c|}{$\begin{array}{l}\text { Materials Research Society of India (MRS-I) } \\
\text { S.K. Joshi, JNCASR, New Delhi }\end{array}$} & \multicolumn{2}{|c|}{$\begin{array}{l}\text { Materials Research Society of Russia (MRS-Russia) } \\
\text { I.V. Gorynin, Prometey Institute }\end{array}$} \\
\hline \multicolumn{2}{|c|}{$\begin{array}{l}\text { Chinese Materials Research Society (C-MRS) } \\
\text { H-D. Li, Tsinghua University }\end{array}$} & \multicolumn{3}{|c|}{$\begin{array}{l}\text { Materials Research Society of Japan (MRS-J) } \\
\text { R-I. Yamamoto, University of Tokyo }\end{array}$} & \multicolumn{2}{|c|}{$\begin{array}{l}\text { Materials Research Society of Taiwan (MRS-T) } \\
\text { L.J. Chen, National Tsing Hua University }\end{array}$} \\
\hline \multicolumn{2}{|c|}{$\begin{array}{l}\text { European Materials Research Society (E-MRS) } \\
\text { G.M. Crean, University College }\end{array}$} & \multicolumn{3}{|c|}{$\begin{array}{l}\text { Materials Research Society of Korea (MRS-Korea) } \\
\text { S-J. Park, Seoul National University }\end{array}$} & \multicolumn{2}{|c|}{$\begin{array}{l}\text { Mexican Materials Research Society (Mexican-MRS) } \\
\text { L.M. Gomez, Instituto de Fisica-Cuernavaca, UNAM }\end{array}$} \\
\hline \multicolumn{2}{|c|}{$\begin{array}{l}\text { Materials Research Society (MRS) } \\
\text { R.J. Nemanich, North Carolina State University }\end{array}$} & & & & & \\
\hline
\end{tabular}




\section{UHV Gate Valves}

Series 10 with "Vulcanized" Gate Seal

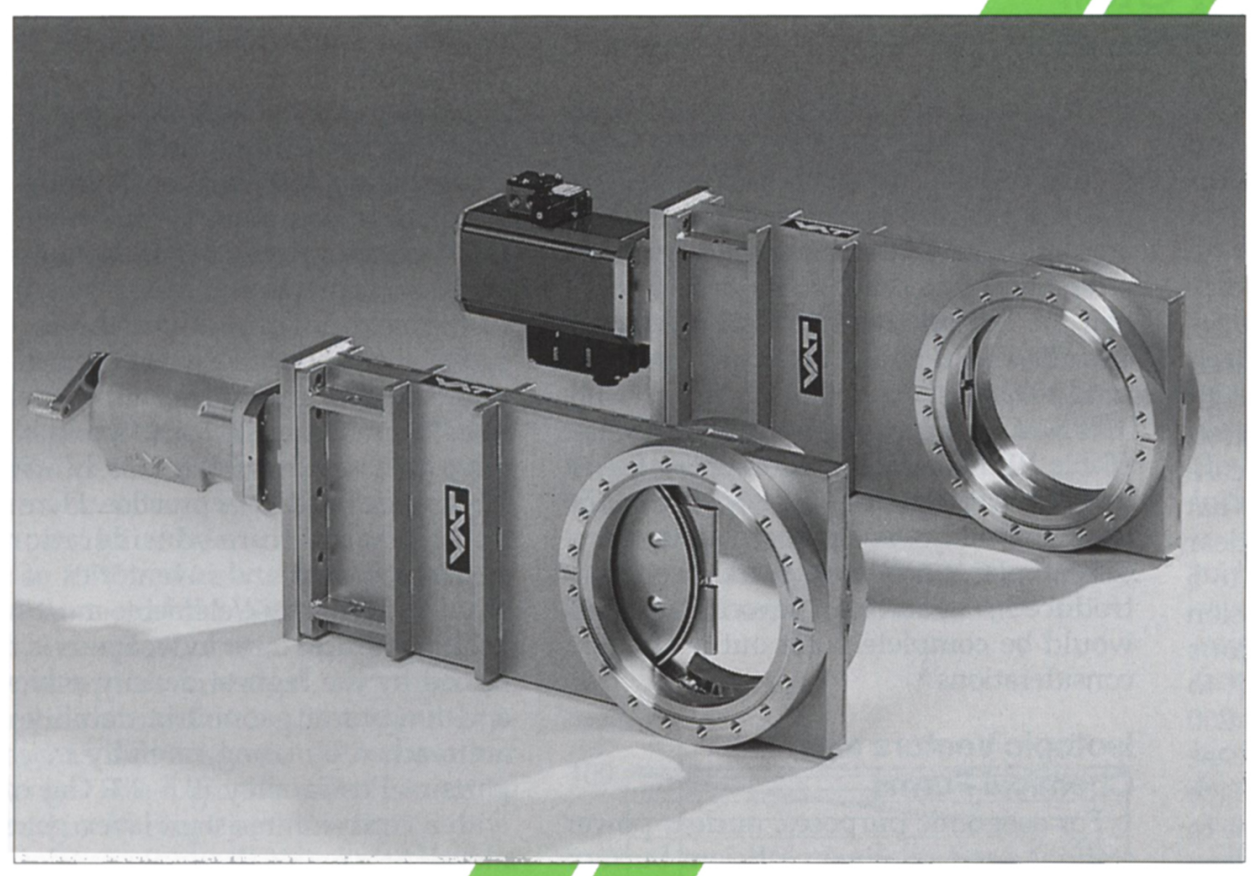

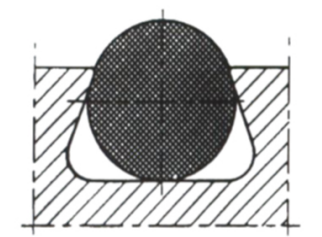

Conventional O-Ring Gate Seal

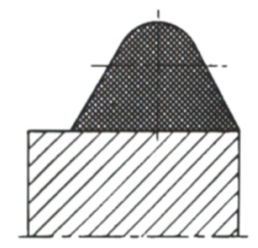

VAT Vulcanized UHV Gate Seal

- One Sealing Line Only - No Trapped Volumes - Higher Lifetime

\section{Request your new Catalog 2000 today}

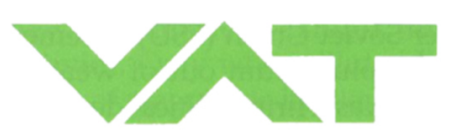

VAT, Inc., 500 West Cummings Park, Woburn, MA 01801 781-935-1446 Fax 781-935-3940 e mail: usa@vatvalve.com http://www.vatvalve.com 